\title{
Algoritmo de Mínimos Cuadrados con Error Codificado para Filtrado Adaptivo
}

\author{
José Velázquez, Juan C. Sánchez y Gerardo Ávalos \\ Instituto Politécnico Nacional, Escuela Superior de Ingeniería Mecánica y Eléctrica, \\ Unidad Culhuacan, Sección de Estudios de Posgrado e Investigación, Av. Santa Ana \#1000, \\ Col. San Francisco Culhuacan, 04430 México, D.F. - México \\ (e-mail: jvelazquez@ieee.org, jcsanchezgarcia@gmail.com, gav88a@hotmail.com)
}

\section{Resumen}

Se presenta el análisis de una variante del algoritmo de mínimos cuadrados para reducir la complejidad de diseño para su implementación en filtros adaptativos digitales. Dicha variante consiste en cambiar la codificación del error en el algoritmo, ya que dicho error es un valor de tipo entero. Los resultados obtenidos en las pruebas realizadas en aplicaciones de filtros adaptativos tales como predictor lineal e identificador de sistema, demuestran que la velocidad de convergencia aumenta. Esto permite que el algoritmo propuesto pueda ser aplicado en filtros adaptativos donde se requiere una velocidad de convergencia alta. Además la modificación propuesta es compatible con los filtros adaptativos existentes, debido a que la codificación del error se puede realizar por separado.

Palabras clave: filtros adaptativos, algoritmo de mínimos cuadrados, error codificado, predictor lineal

\section{Least Mean Square Algorithm with Codified Error for Adaptive Filters}

\begin{abstract}
Analysis of a modified least mean square algorithm to reduce its design complexity for digital adaptive filter implementation, is presented. Such a modification consists of changing the error codification of the algorithm, because such error is an integer number. Results obtained during the testing of the method in applications to adaptive filters such as linear prediction and system identifier, show that the speed convergence increases. This allows the algorithm to be applied to adaptive filter for which high speed is required. Also, the proposed modification is compatible with existing adaptive filters, since error codification can be separately done.
\end{abstract}

Keywords: adaptive filters, least mean square algorithm, codified error, linear predictor 


\section{INTRODUCCIÓN}

Debido al gran desarrollo de los sistemas de comunicaciones, los problemas que se presentan aumentan en la misma medida, lo que incrementa la complejidad de diseño de los sistemas para evitar el menor número de errores en la transmisión de información. Por ejemplo, cancelación de eco, ruido aditivo, interferencia íntersímbolo, son problemas considerables que se presentan a menudo en sistemas de comunicaciones digitales; su solución está en el uso de filtros adaptivos. Los filtros adaptivos son sistemas que adaptan sus parámetros de acuerdo a las variables que se presentan en su entorno y tienen una estructura como la que se muestra en la Fig. 1, en donde se puede ver, que son sistemas de cuatro terminales con una señal de entrada $x$, la señal deseada a la cual se quiere aproximar la respuesta del filtro $d$, además genera una señal de salida $y$ con una señal de error $e$ igual a la diferencia entre la señal deseada y de salida del filtro adaptivo. La señal de error se emplea como referencia para adaptar los parámetros del filtro (Carusone y Johns, 2000).

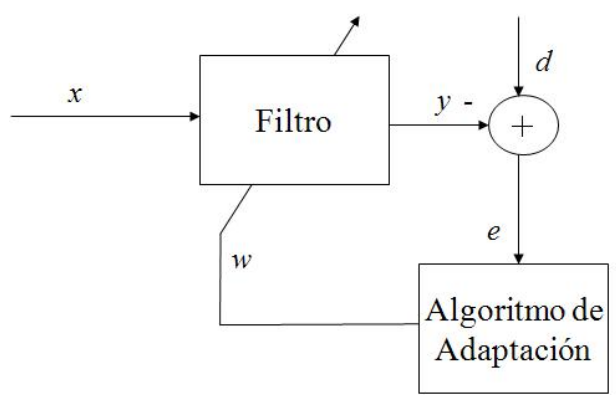

Fig. 1: Estructura general de los filtros adaptivos.

La eficiencia de los filtros adaptivos depende principalmente de la técnica de diseño utilizada y el algoritmo de adaptación. Los filtros adaptivos pueden ser diseños analógicos (Carusone y Johns, 2000; Pérez et al., 2001; Lee y Razavi, 2001), digitales o mixtos los cuales presentan sus ventajas y desventajas, por ejemplo, los filtros analógicos son de bajo consumo de potencia y respuesta rápida, pero presentan problemas de desbalanceo (offset), el cual afecta el funcionamiento del algoritmo de adaptación (Shoval et al., 1995). Los filtros digitales son libres del desbalanceo y ofrecen una respuesta de mayor precisión, pero son de gran complejidad debido a las operaciones de punto flotante. Los diseños mixtos (Bhupendra et al., 1979a, 1979b; Figueroa et al., 2004), presentan problemas de desacoplamiento entre los bloques analógicos y digitales. Estas técnicas realizan el procesamiento de la señal en el dominio del tiempo, pero también existen filtros adaptivos que trabajan en el dominio de la frecuencia (Jackson, 1998; Bogucka y Wesolowski, 2000; Van Acker et al., 2001), los cuales realizan filtrado adaptivo en sub-bandas con buena respuesta, pero el diseño de estos filtros requieren de procesadores digitales de señales. Otras propuestas realizan una modificación directa a la estructura del filtro para aumentar la eficiencia en la convolución (Chen et al., 1996).

Los algoritmos de adaptación más conocidos, son el algoritmo de mínimos cuadrados recursivos (RLS - Recursive Least Square), y el algoritmo de mínimos cuadrados (LMS - Least Mean Square), en donde el algoritmo RLS ofrece mayor velocidad de convergencia con respecto al algoritmo LMS, pero en cuanto complejidad computacional, el algoritmo LMS tiene la ventaja. Por su simplicidad computacional, el algoritmo LMS es el que tiene mayor uso en el diseño e implementación de filtros adaptivos digitales (Avalos et al., 2007).

\section{ALGORITMO LMS Y SUS VARIANTES}

El algoritmo LMS digital se basa en la búsqueda del gradiente del error cuadrático de acuerdo con la ecuación (1).

$w_{k}(T+1)=w_{k}(T)+\mu e(T) x_{k}(T)$

en donde $w_{k}(T)$ es el vector de pesos actual, $w_{k}(T+1)$ es igual al vector de pesos siguiente, $x_{k}(T)$ es el vector de retardos con las muestras de la señal de entrada, $e(T)$ corresponde al error instantáneo del 
filtro y $\mu$ es el factor de convergencia del filtro. El factor de convergencia $\mu$, determina la velocidad de convergencia, el cual es directamente proporcional a la velocidad de convergencia e inversamente proporcional al error cuadrático medio mínimo, por lo tanto, existe un compromiso entre velocidad de convergencia y error cuadrático promedio mínimo.

Para evitar la divergencia, el factor de convergencia debe cumplir lo estipulado en la ecuación (2) (Chen et al., 1996):

$0<\mu<\frac{1}{N \overline{x^{2}(T)}}$

donde $\overline{x^{2}(T)}$ denota el valor cuadrático medio de la potencia de la señal $x(T)$ estimado con un número reducido de muestras y $N$ es el número de elementos de retardo del filtro.

Se han realizado algunas modificaciones al algoritmo LMS con la finalidad de reducir más su complejidad computacional, pero a costa de la velocidad de convergencia y del error mínimo. Estas modificaciones han considerado el signo o los signos de la señal de entrada y del error como se muestra en las ecuaciones (3), (4) y (5).

$$
\begin{aligned}
& w_{k}(T+1)=w_{k}(T)+\mu \operatorname{sign}[e(T)] x_{k}(T) \\
& w_{k}(T+1)=w_{k}(T)+\mu \operatorname{sign}\left[x_{k}(T)\right] e(T) \\
& w_{k}(T+1)=w_{k}(T)+\mu \operatorname{sign}\left[x_{k}(T)\right] \operatorname{sign}[e(T)]
\end{aligned}
$$

Otras modificaciones al algoritmo LMS, consisten en cuantizar las señales de entrada y de error a la potencia de dos más cercana. Estas propuestas se muestran en las ecuaciones (6) conocida como Log-LMS y en (7) también conocida como Log-Log LMS (Shivaling et al., 1997).

$$
\begin{aligned}
& w_{k}(T+1)=w_{k}(T)+\mu Q[e(T)] Q\left[x_{k}(T)\right] \\
& w_{k}(T+1)=w_{k}(T)+Q\left[\mu Q[e(T)] Q\left[x_{k}(T)\right]\right]
\end{aligned}
$$

Las modificaciones mostradas en las ecuaciones anteriores, presentan una velocidad de convergencia lenta, debido a que pierden información del incremento del error.

\section{ALGORITMO LMS CON ERROR CODIFICADO}

Con los algoritmos propuestos anteriormente, se propone realizar una modificación al algoritmo de adaptación LMS, con el propósito de reducir el número de operaciones de punto flotante y con ello tener un algoritmo más simple de implantar en un filtro adaptivo digital.

La propuesta consiste en modificar el error de tal manera que el algoritmo detecta un error mayor al error instantáneo, con lo cual se logra aumentar la velocidad de convergencia, sin olvidar la reducción de la complejidad del algoritmo para su posible implantación en un filtro digital, disminuyendo las operaciones de punto flotante empleando números enteros. Entonces con las consideraciones realizadas, se propone realizar la codificación del error, es decir, representar el error instantáneo mediante un valor entero, con lo cual el error aparente tiene un valor mayor y además no se pierde información significativa del incremento del error, lo cual es importante en la velocidad de convergencia. Entonces el algoritmo LMS con error codificado (ECLMS) queda como se muestra en la ecuación (8) (Velázquez, 2004).

$w_{k}(T+1)=w_{k}(T)+\mu C[e(T)] x_{k}(T)$ 
Analizando la ecuación (8), el número de operaciones de punto flotante se reduce, debido a que el error codificado es un valor entero, el cuál al multiplicarse con la muestra de la señal de entrada que también tiene un valor entero, el resultado no depende de una representación de punto flotante, lo cual complica el diseño digital. Recordando que el factor de convergencia requiere representarse como un valor de punto flotante, se puede simplificar su representación, empleando una notación de potencia de dos, en donde el valor del exponente indica el número de lugares en que se tiene que recorrer el punto decimal del resultado del producto entre error instantáneo y la muestra de la señal de entrada.

Otro aspecto notable con la codificación del error, es el hecho de que no se altera el algoritmo de adaptación, debido a que la codificación del error se puede efectuar de forma externa al algoritmo. Entonces la adecuación del algoritmo en sistemas digitales existentes no se vería afectado considerablemente, ya que solo basta agregar el codificador.

Para realizar matemáticamente la codificación del error, se requiere efectuar su cuantización y asignarle un código, empleando lo mostrado en la ecuación (9).

$C[e(T)]=\operatorname{round}\left(\frac{e(T)}{\operatorname{Res}}\right)$

en donde $e(T)$ es el error actual del filtro, Res la resolución del codificador y round redondea el resultado de la división entre ambos. Entonces el error obtenido de (9), es un número entero al cual se le asigna un código binario.

La resolución del codificador se obtiene empleando (10).

Res $=\frac{E r r_{\text {máx }}}{2^{n}-1}$

donde $E r r_{\max }$ es el error máximo probable y $n$ corresponde al número de bits de codificación. Debido a que se desconoce el error máximo, se recurre a realizar una estimación a partir de la amplitud máxima de la señal deseada o de la señal de entrada del filtro adaptivo.

Como se puede ver, la modificación propuesta no afecta a la estructura del filtro y tampoco al algoritmo, por lo cual el algoritmo ECLMS puede ser compatible con los sistemas de filtrado existentes. Otro punto importante es el hecho de que se reducen operaciones de punto flotante, debido a que el error codificado es un número entero, ocasionando que una parte de la operación del algoritmo se reduzca a operaciones de suma y desplazamiento o la utilización de memoria, lo cual es más práctico en diseños digitales (Ávalos et al., 2007).

\section{Efectos de la codificación del error}

En esta sección se analizan cuales son los efectos de la codificación del error sobre el algoritmo LMS y así obtener las condiciones necesarias para que funcione adecuadamente sin alterar la respuesta de la propuesta realizada con respecto al algoritmo LMS convencional. Como primer efecto se puede detectar el aumento en la velocidad de convergencia, dado que el error inicial que observará el algoritmo será grande haciendo que el algoritmo aumente el tamaño de los pesos aumentando la velocidad de convergencia.

Otro aspecto relevante es el comportamiento del algoritmo conforme avanza el proceso de adaptación. Cuando el proceso de adaptación avanza, la magnitud del error disminuirá, y con la codificación del error, el algoritmo ECLMS tendrá un comportamiento similar al algoritmo LMS con error de signo, debido a que la magnitud del error codificado puede ser igual a uno o cero, siempre y cuando la magnitud del error instantáneo, se encuentre en la condición mostrada en la ecuación (11) (Velásquez et al., 2007). 


$$
\frac{1}{2} \operatorname{Res} \leq|e(T)|<\frac{3}{2} \operatorname{Res}
$$

De la ecuación (11) se puede detectar que al seleccionar un valor de resolución grande, entonces el algoritmo EC-LMS requiere de unas cuantas iteraciones para comportarse como la variante de signo, pero en consecuencia se afecta en la velocidad de convergencia y en el error cuadrático medio mínimo.

Cuando el valor de la resolución es demasiado pequeño, entonces la cantidad de información que se pierde es menor, por lo tanto la velocidad de convergencia es mayor. La resolución es inversamente proporcional al número de bits de codificación, entonces para obtener una resolución grande, se requiere de un número de bits reducido y para reducir la resolución es necesario un mayor número de bits, pero se debe tener cuidado en elegirlo correctamente, ya que una cantidad de bits grande puede hacer que el algoritmo llegue a divergir.

Para evitar la divergencia del algoritmo LMS al incrustar la codificación del error, se sustituye las operaciones de la codificación del error explicadas anteriormente, en la ecuación (8) para obtener (12) donde $Q[e(T)]$ es la cuantización del error para realizar la codificación.

$w_{k}(T+1)=w_{k}(T)+\mu \frac{Q[e(T)]}{\operatorname{Res}} x_{k}(T)$

De la ecuación (12), se observa que existe una relación entre el valor de la resolución con el factor de convergencia, entonces para facilitar el análisis, se realiza el cambio de variable en el factor de convergencia $\mu$ para obtener (13)

$$
w_{k}(T+1)=w_{k}(T)+\mu^{\prime} Q[e(T)] x_{k}(T)
$$

donde:

$\mu^{\prime}=\frac{\mu}{\operatorname{Res}}$

por lo tanto el factor de convergencia resultante $\mu^{\prime}$ es inversamente proporcional a la resolución.

El factor de convergencia debe satisfacer (2), entonces al realizar la codificación del error se debe considerar la resolución, entonces sustituyendo (14) en (2) se obtiene (15):

$0<\mu^{\prime} \leq \frac{1}{\mathrm{Nx}^{2}(T)}$

sustituyendo (14) en (15) y despejando el factor de convergencia se obtiene (16):

$0<\mu \leq \frac{\operatorname{Res}}{\mathrm{Nx}^{2}(T)}$

sustituyendo la resolución de la ecuación (10) en (16) se obtiene:

$0<\mu \leq \frac{\text { error }_{\max }}{\mathrm{Nx}^{2}(T)\left(2^{n}-1\right)}$ 
lo cual indica que el factor de convergencia para el algoritmo LMS con error codificado, además de lo expuesto en la ecuación (2), es inversamente proporcional al número de combinaciones posibles con $n$ número de bits de codificación. Por lo tanto, al aumentar el número de bits en la codificación del error, entonces tendrá que disminuir el factor de convergencia. Es por ello que es necesario tener cuidado en la selección del número de bits para la codificación del error, el cuál estará relacionado con el factor de convergencia.

\section{RESULTADOS Y DISCUSIÓN}

En esta sección se presentan los resultados obtenidos, con el propósito de realizar un análisis comparativo de la modificación propuesta. Los resultados que se muestran, realizan la comparación con respecto al algoritmo LMS convencional y el algoritmo LMS sobreadaptado cuando el filtro adaptivo lleva a cabo la predicción lineal y la identificación de sistemas, además se realiza el análisis de comportamiento del número de bits de codificación y la resolución del codificador.

\section{Predictor lineal}

La predicción lineal es una técnica de estimación espectral, la cual se emplea para modelar procesos aleatorios correlacionados, con el objetivo de encontrar la representación paramétrica de estos procesos. Los resultados de la comparación del algoritmo LMS contra el algoritmo modificado, se muestra en la Fig. 2, los cuales se obtuvieron utilizando Matlab ${ }^{\mathrm{TM}}$, empleando un filtro con estructura transversal, el mismo factor de convergencia y considerando al error máximo del $90 \%$ del valor pico de la señal de entrada.

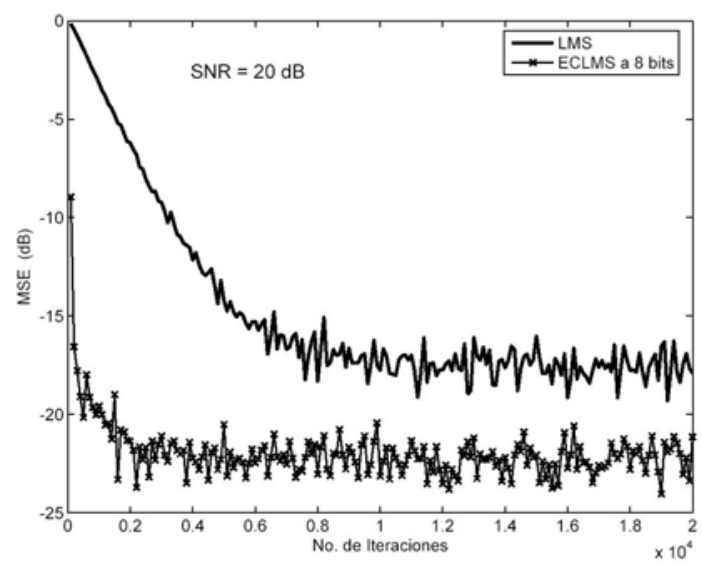

Fig. 2: Error cuadrático medio del algoritmo LMS contra ECLMS.

En los resultados mostrados en la Fig. 2, se puede observar que el error cuadrático medio (MSE Mean Square Error) para el algoritmo ECLMS, converge mucho más rápido con respecto al algoritmo LMS convencional, además de que no se empeora el error cuadrático mínimo en ECLMS. El aumento de la velocidad de convergencia con ECLMS se debe a que de manera indirecta se está afectando al factor $\mu$, debido a que inicialmente el error es grande y por lo tanto el error codificado corresponde a un valor grande, pero como avanza el proceso de adaptación, el error va disminuyendo hasta tener un comportamiento similar a la variante de signo del algoritmo LMS, debido a que el error codificado tendrá valores de uno o cero.

Otra prueba realizada, fue cambiar el número de bits de codificación de la señal de error, empleando las mismas condiciones de operación que en el experimento anterior. Con este experimento se obtiene la relación que existe entre el número de bits, la velocidad de convergencia y el error mínimo. Los resultados obtenidos se muestran en la Fig. 3.

Los resultados mostrados en la Fig. 3, demuestran que a mayor número de bits, la velocidad de convergencia es mayor, pero cuando el número de bits es mayor a ocho, el error cuadrático medio diverge debido a que no se está cumpliendo con la condición estipulada en la ecuación (17). 
Además, al realizar la codificación con un solo bit, el algoritmo propuesto es similar a la variante del algoritmo LMS cuando solo se considera el signo de la señal de error.

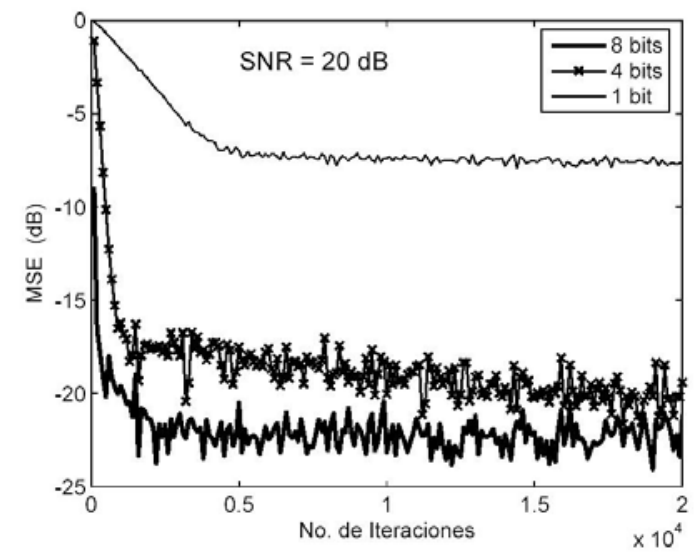

Fig. 3: LMS con error codificado empleando diferente número de bits de codificación.

Cuando el algoritmo EC-LMS converge, el valor del error codificado puede ser igual a cero, lo cual se puede aprovechar en la reducción de operaciones matemáticas a efectuar, porque cuando es igual a cero, entonces el valor de los pesos se mantiene con el mismo valor y por lo tanto se reduce el número de operaciones a realizar, lo cual es una ventaja en el tiempo de procesamiento en sistemas digitales.

También es importante saber como poder determinar el error máximo probable, las pruebas anteriores se hicieron considerando que el error máximo probable, es igual al $90 \%$ de la amplitud máxima de la señal de entrada. Cambiando la resolución con respecto a otros valores máximos de la señal de entrada, se obtienen los resultados que se muestran en la Fig. 4.

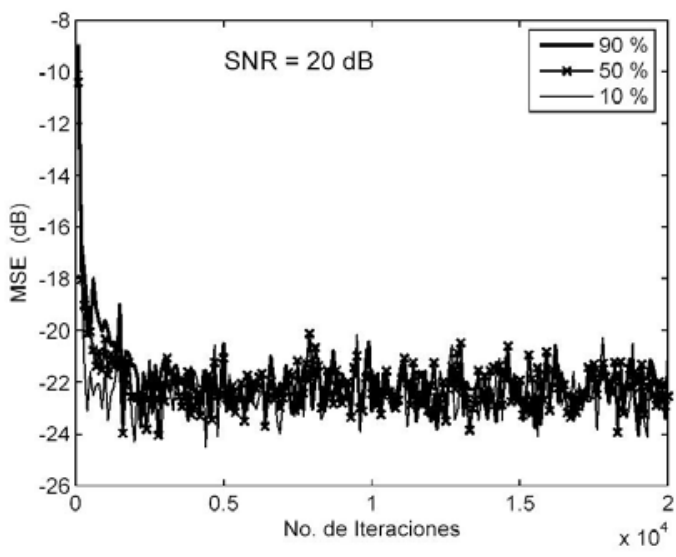

Fig. 4: LMS con error codificado con diferentes valores de resolución.

En la Fig. 4, se observa que la velocidad de convergencia no se ve afectada cuando se cambia la resolución del codificador, solo cambia ligeramente el error mínimo cuando el error máximo probable disminuye. Esto sucede cuando el error es menor al valor del error máximo, pero cuando el error es mayor al valor del error máximo programado, entonces el algoritmo ECLMS generará un error cuadrático medio mayor o en el peor de los casos se puede presentar la divergencia.

Otro análisis interesante, es observar la relación que existe entre la resolución y el número de bits de codificación. Se realizó la prueba de modificando el valor de la resolución y empleando un bit de codificación obteniéndose los resultados de la Fig. 5.

La gráfica de la Fig. 5, indica que la velocidad de convergencia y el error cuadrático medio mínimo en el algoritmo ECLMS, no solamente depende en gran medida del número de bits de codificación, 
también depende de la resolución utilizada. Entonces, si requiere de utilizar un menor número de bits de codificación, entonces el error máximo que se debe considerar en la resolución debe ser menor también.

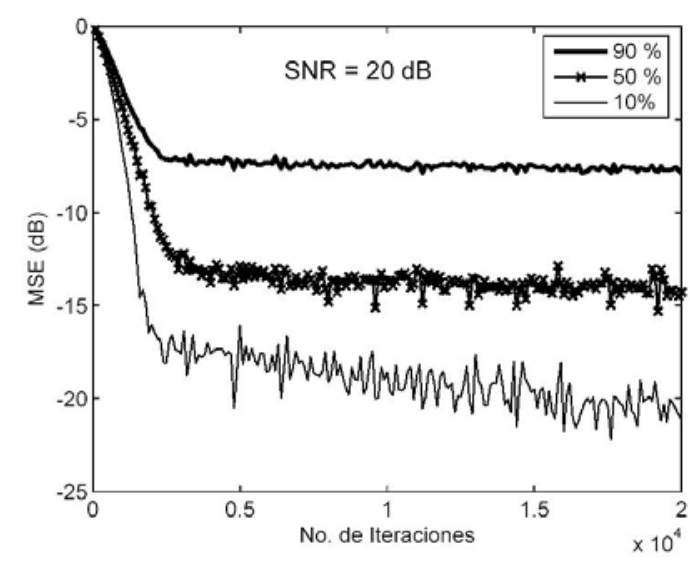

Fig. 5: Análisis de Resolución y número de bits.

\section{Identificador de sistema}

La identificación de sistemas, es una herramienta usada para obtener los parámetros de sistemas cuyo modelo se desconoce, para ello se aplica la misma señal de entrada $x(T)$ al sistema desconocido y al identificador de sistema, entonces la señal deseada será la respuesta del sistema desconocido. El sistema desconocido usado, es un filtro transversal con pesos generados de manera aleatoria, en donde el número de elementos de retardo es el mismo en el sistema desconocido y en el filtro adaptivo.

Al igual que el predictor lineal, se midió el error cuadrático medio, comparando el algoritmo ECLMS, contra el algoritmo LMS convencional. Los resultados obtenidos se muestran en la Fig. 6, en donde se observa que el algoritmo LMS con error codificado, requiere de un número menor de iteraciones para encontrar el error mínimo local que obtiene el algoritmo LMS convencional. Esto se debe a lo explicado en los resultados obtenidos en la predicción lineal, al realizar la codificación del error, se afecta indirectamente al factor de convergencia hasta que converge el algoritmo.

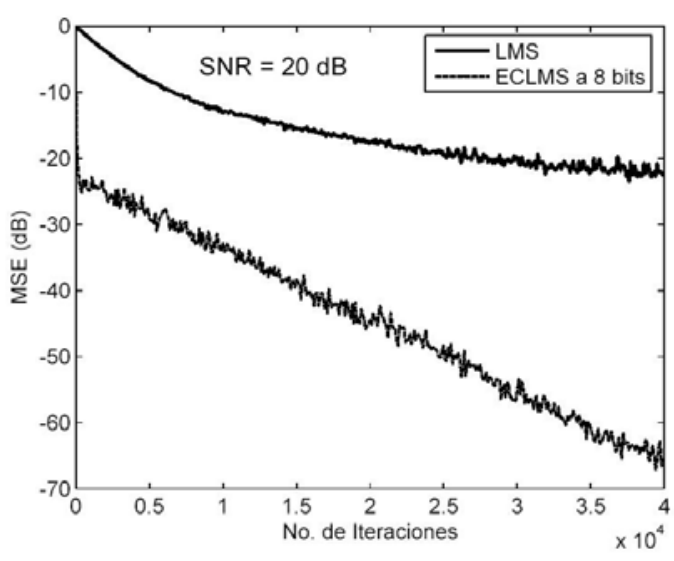

Fig. 6: Error cuadrático medio del algoritmo LMS contra ECLMS.

Al igual que en el predictor, se cambió el número de bits de codificación. Los resultados se muestran en la Fig. 7, en donde se puede comprobar la proporcionalidad existente entre la velocidad de convergencia y el número de bits empleados en la codificación del error, pero sin perder la importancia de cumplir la condición de la ecuación (17) para evitar la divergencia. 


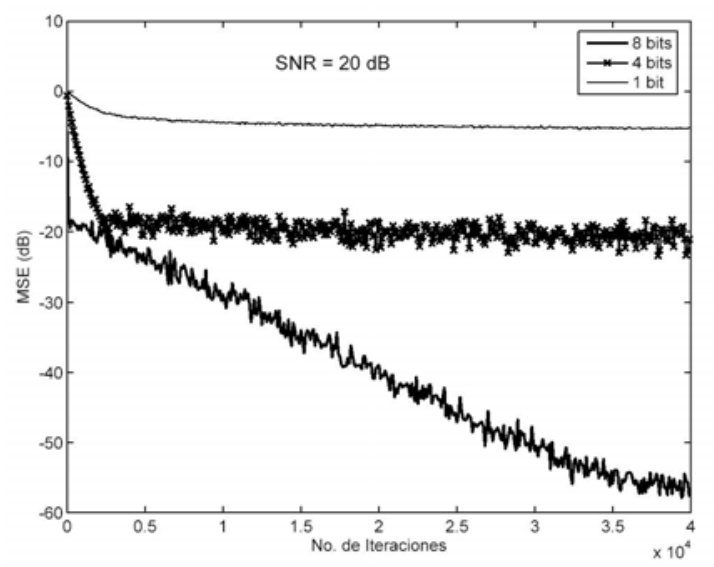

Fig. 7: LMS con error codificado empleando diferente número de bits de codificación.

Otro punto aspecto importante a revisar, es el efecto de la resolución que se emplea en el codificador, para el caso de identificador de sistema. La Fig. 8, muestra los resultados obtenidos codificando a ocho bits, pero modificando la resolución para observar su efecto en el error cuadrático medio. De los resultados mostrados en la Fig. 8, se puede ver que la resolución del codificador no afecta en la velocidad de convergencia del algoritmo LMS con error codificado. Al disminuir la resolución se logra reducir el error cuadrático medio, pero cuando la resolución es muy baja, el mínimo local del error cuadrático es mayor.

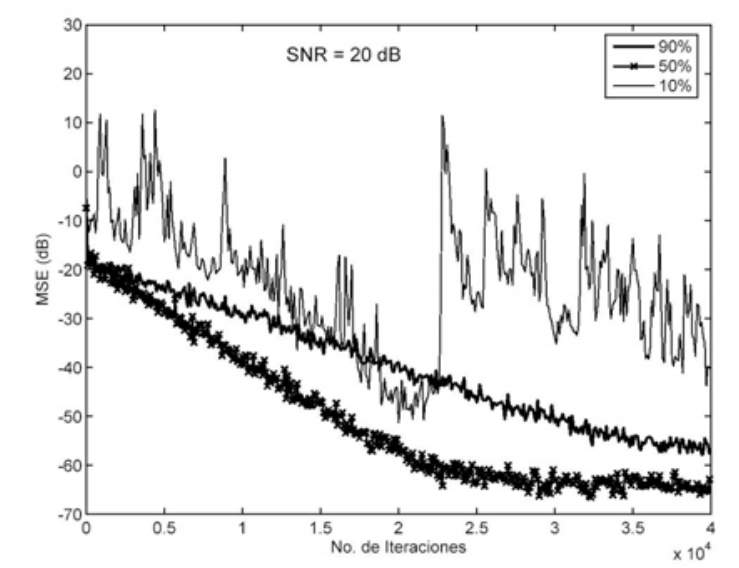

Fig. 8: ECLMS con diferentes valores de resolución.

Para observar la relación que existe entre la resolución del codificador y el número de bits de codificación, se realizó la simulación cambiando el valor máximo del error y codificando el error con un bit, el resultado de esta prueba se muestra en la Fig. 9, en donde se obtiene un MSE mínimo, cuando se emplea como amplitud máxima del error igual al 10\% de la amplitud máxima de la señal de entrada.

\section{Identificador de sistema con re-uso de datos}

Una variante del algoritmo LMS para aumentar la velocidad de convergencia, es el uso del mismo vector de datos para reajustar el vector de pesos durante $j$ iteraciones mas como lo muestra la ecuación (18), esta variante se conoce también como el algoritmo LMS sobreadaptado (Roy y Shynk, 1989).

$w_{k}(T+j)=w_{k}(T+j-1)+\mu \alpha_{k}(T) e(T+j-1)$

Como se observa en (18), se realiza el recalculo del vector de pesos sin realizar el corrimiento en el vector de datos de la señal de entrada. Esta modificación no es reciente, y está pensada para aplicarse en sistemas con alta velocidad de procesamiento. A continuación se realiza la comparación 
entre el algoritmo ECLMS y LMS con re-uso de datos, o también conocida como LMS sobreadaptado, empleando las mismas condiciones de trabajo para los dos algoritmos y observar su rendimiento con la medición del error cuadrático promedio. En la Fig. 10, se observa la comparación entre el algoritmo LMS sobreadaptado con cinco iteraciones adicionales, ECLMS con 8 bits y ECLMS sobreadaptado con 8 bits, detectándose la diferencia en la velocidad de convergencia y el valor mínimo del error cuadrático medio.

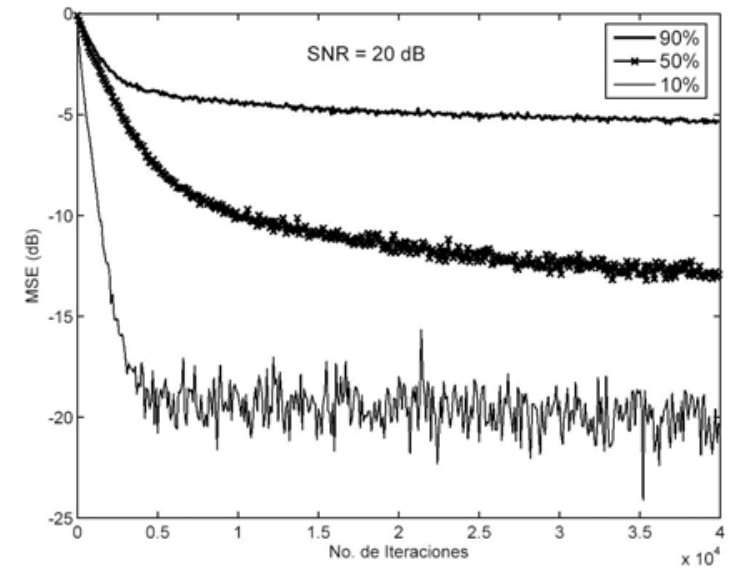

Fig. 9: Análisis de Resolución y número de bits de codificación.

Los resultados de la Fig. 10, se obtuvieron empleando el mismo factor de convergencia y la señal de entrada con las mismas características, con el fin de obtener una comparación en el comportamiento del algoritmo LMS y ECLMS en diferentes aplicaciones y variantes.

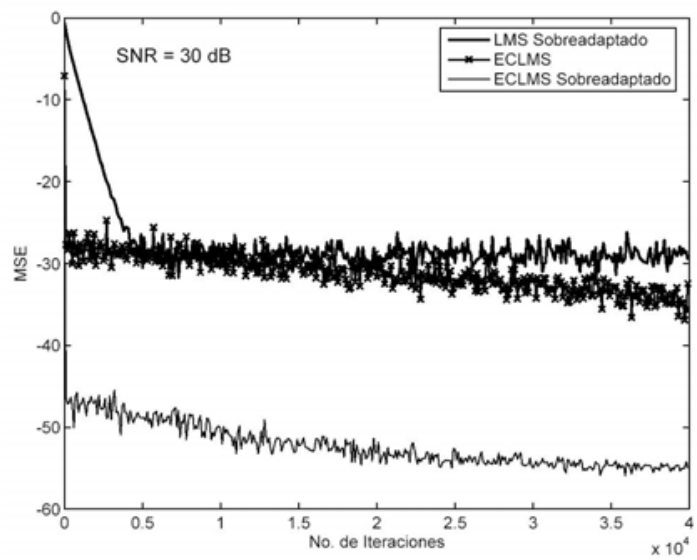

Fig. 10: MSE para LMS sobreadaptado y ECLMS.

Al aplicar la sobreadaptación en el algoritmo ECLMS, se obtiene un error cuadrático medio mínimo local menor, lo cual es un beneficio en la reducción de la potencia del error, debido al recalculo de los pesos en cada iteración normal del algoritmo LMS convencional.

Una prueba adicional realizada, es realizando la comparación entre el algoritmo ECLMS cuando se adapta utilizando ocho bits en la codificación del error y un ECLMS adaptado con ocho bits y sobreadaptado con diez bits en la codificación del error, obteniéndose el resultado mostrado en la Fig. 11. Los resultados de la Fig. 11, muestran una disminución del error cuadrático medio mínimo, cuando se utiliza un mayor número de bits en el algoritmo ECLMS adaptado con ocho bits y sobreadaptado con diez bits. Esto se debe a que en la sobreadaptación, con la codificación del error con un mayor número de bits permite tener mayor cantidad de información sobre la magnitud del error, reflejándose en el nivel del error cuadrático medio. Esto da pie a realizar combinaciones con el número de bits en la codificación del error para adaptar y sobreadaptar y con ello obtener mejores resultados. 


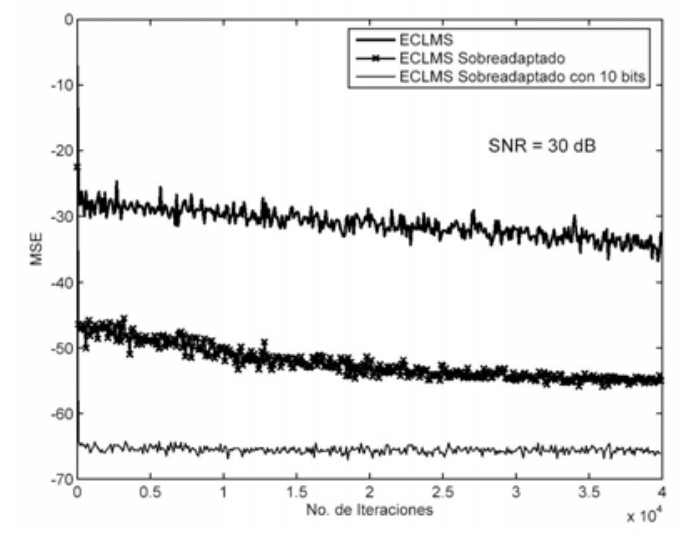

Fig. 11: Comparación del algoritmo ECLMS Sobreadaptado.

\section{CONCLUSIONES}

Se presentó una modificación al algoritmo LMS, con el objetivo de aumentar su velocidad de convergencia y disminuir el número de operaciones de punto flotante. Esto permite una implementación menos compleja en filtros adaptivos digitales.

La modificación propuesta no afecta a la estructura del filtro adaptivo y tampoco al algoritmo de adaptación, debido a que la codificación del error se realiza por separado, por lo tanto, el algoritmo ECLMS es compatible con los filtros adaptivos digitales existentes.

La reducción de la complejidad del filtro adaptivo, se puede mejorar con la buena elección del número de bits de codificación y la resolución del codificador.

Los resultados mostrados con la sobreadaptación del algoritmo ECLMS, demuestran la posibilidad de poder obtener una mejora en la disminución de la potencia del error y al conjuntarse con la combinación del número de bits en la codificación del error, se puede disminuir la complejidad de implantación de los filtros adaptivos digitales.

Otro aspecto importante, es que la rápida velocidad de convergencia que se logra obtener con la codificación del error, permite emplear este algoritmo en sistemas de comunicación de alta velocidad, por ejemplo ADSL, en donde se requiere que los filtros adaptivos tengan una rápida velocidad de convergencia.

\section{REFERENCIAS}

Ávalos J.G, J.M. González, J. Velázquez y J.C. Sánchez; "Implementación del algoritmo LMS con error codificado en el DSP TMS320C6713", Congreso Nacional de Ingeniería Electromecánica y de Sistemas, Ciudad de México, México, Noviembre 26-30 (2007).

Bhupendra K.A., A.C. Miles y H. Chong; "A new adaptive algorithm and its implementation in MOS LSI"; IEEE Journal of Solid State Circuits, 14 (4), 747-753 (1979a).

Bhupendra K.A., A.C. Miles y H.C. Chong; "A Sampled Analog MOS LSI Adaptive Filter", IEEE Journal Of Solid-State Circuits, 14, (1), 148-154 (1979b).

Bogucka H. y K. Wesolowski; "Frequency-Domain Echo Cancellation in Digital Multicarrier Modulation Systems"; IEEE Transactions on Communications; 48 (2), 333-342 (2000). 
Carusone, A. y D.A. Johns; "Analogue adaptive filters: past and present"; Circuits, Devices and Systems, IEEE Proceedings; 147, 82-90 (2000).

Chen S, Y. Kao y K. Tsai; "A New Efficient LMS Adaptive Filtering Algorithm"; IEEE Transactios on Circuits and Systems -II, 43 (5), 372-378 (1996).

Figueroa M., S. Bridges, D. Hsu y C. Diorio; "A 19.2 GOPS Mixed-Signal Filter With Floating-Gate Adaptation", IEEE Journal of Solid State Circuits, 39 (7), 1196-1201 (2004).

Jackson A.; "ADSL For High-Speed Broadband Data Service"; Aerospace Conference, 1998. Proceedings., IEEE, 4, 451-465 (1998).

Lee Tai-Cheng y B. Razavi; "A 125 Mixed-Signal Echo Canceller for Gigabit Ethernet on Copper Wire"; IEEE Journal of Solid-State circuits, 36 (3), 366-373 (2001).

Pérez Meana H., M.N. Miyatake, L. Niño de Rivera y J. Sánchez García; "Desarrollos recientes en sistemas adaptables analógicos"; Revista Científica; 5 (3), 113-129 (2001).

Roy S. y J.J. Shynk; "Analysis of the Data-Reusing LMS Algorithm", Circuits and Systems, (2), 11271130 (1989).

Shivaling S. Mahant-Shetti, S. Hosur y A. Gatherer; "The Log-Log LMS Algorithm" Acoustics, Speech, and Signal Processing, 1997. 3, 2357-2360 (1997).

Shoval A., D.A. Johns y W. Martin Snelgrove; "Comparison of DC Offset Effects in Four LMS Adaptive Algorithms"; IEEE Transactions on Circuits and Systems-II: Analog and Digital Signal Processing; 42 (3), 176-185 (1995).

Van Acker K., G. Leus y M. Moneen; "Per Tone Equalization for DMT-Based Systems"; IEEE Transactions on Communications, 49 (1), 109-119 (2001).

Velázquez López J, J.G. Ávalos Ochoa, J.C. Sánchez García y L. Díaz Hernández; "Comparación del Algoritmo de Mínimos Cuadrados Promediados con Error Codificado, con Algoritmos de Paso Variable", Congreso Nacional de Ingeniería Electromecánica y de Sistemas, Ciudad de México, México, Noviembre 26-30 (2007).

Velázquez López J., J.C. Sánchez García y H. Pérez Meana; "Algoritmo LMS con error codificado"; CAS-Tour/ICED, Veracruz, México Nov 16-19. (2004). 